\title{
RÉGIMEN JURÍDICO DEL PROFESORADO UNIVERSITARIO VINCULADO A LAS INSTITUCIONES SANITARIAS: NECESIDAD DE REVISIÓN
}

\author{
MIRIAM CUETO PÉREZ1 \\ Universidad de Oviedo \\ mcuetop@uniovi.es
}

Cómo citar/Citation

Cueto Pérez, M. (2017).

Régimen jurídico del profesorado universitario vinculado a las instituciones sanitarias: necesidad de revisión. Revista de Administración Pública, 204, 313-341. doi: https://doi.org/10.18042/cepc/rap.204.11

\section{Resumen}

Este trabajo analiza el régimen jurídico del profesorado universitario vinculado a las instituciones sanitarias, régimen jurídico que no ha sido adaptado a la nueva realidad del EEES y que se encuentra ampliamente superado. En el trabajo se apuntan aquellos aspectos que debieran ser modificados por el legislador, ante la problemática surgida en los últimos tiempos, en relación con numerosos aspectos de sus funciones en la formación de los nuevos profesionales sanitarios.

\section{Palabras clave}

Profesor universitario; instituciones sanitarias; régimen jurídico.

Profesora titular de Derecho Administrativo (acreditada catedrática). Este trabajo tiene lugar en el desarrollo del proyecto de investigación MINECO 16-DER201568256-R «Respuestas jurídicas ante los riesgos derivados de las transformaciones en la Administración: demandas sociales y protección del e-ciudadano». 


\section{Abstract}

This work deals with the legal provisions on University professors joined to Health institutions. These legal provisions have become obsolete and need to be updated to afford the new reality encouraged by EHEA. In this work, aspects and contents that should be changed by legislative authority are pointed out, specially some aspects related to their functions in order to academic qualifications of future Health professionals.

\section{Keywords}

University professor; Health institutions; legal provisions. 


\section{SUMARIO}

I. INTRODUCCIÓN. II. RECORRIDO POR LA NORMATIVA REGULADORA. III. ASPECTOS JURÍDICOS A TENER EN CUENTA ANTE UNA NUEVA REGULACIÓN DE LAS PLAZAS VINCULADAS: 1. Diferencias en el acceso a las plazas de cuerpos docentes universitarios y a los puestos directivos asistenciales. 2. Falta de equivalencia entre el número de profesores y los puestos de responsabilidad en el ámbito asistencial. 3. Incremento de las tareas docentes con el EEES y su compatibilidad con los puestos de responsabilidad asistencial. 4. Dedicación, incompatibilidades y jubilación. IV. REFLEXIONES FINALES.

\section{INTRODUCCIÓN}

La necesidad de las universidades de garantizar una formación al máximo nivel en las profesiones sanitarias tituladas, y especialmente en los estudios de Medicina, tanto en el ámbito teórico como en el asistencial, llevó en su día a un modelo docente que se ha sustentado en los últimos treinta años en los llamados profesores universitarios vinculados ${ }^{2}$. La formación clínica de los estudios de Medicina tiene una especial trascendencia en unas enseñanzas en las que el contacto con el hospital resulta imprescindible para los futuros profesionales. Los profesores vinculados han sido los encargados de protagonizar el necesario acercamiento entre el ámbito académico y el ámbito hospitalario, desarrollando dos actividades inseparables en la docencia universitaria de las áreas clínicas de Ciencias de la Salud: la asistencial y la docente. Dos actividades a las que se ha ido sumando irremediablemente una tercera de peso creciente, que acompańa siempre la labor de los profesores universitarios, que no es otra que la labor investigadora, pieza clave en estos momentos para la obtención de las acreditaciones previas al concurso que deben superar los aspirantes a formar parte de los cuerpos docentes universitarios.

2 La Ley 44/2003, de 21 de noviembre, de ordenación de las profesiones sanitarias, dedica los arts. 12 a 36 a la formación de los profesionales sanitarios. 
Desde la temprana STS de 3 de julio de $1989^{3}$, en la que se clarifican algunos aspectos del RD 1558/1986, de 28 de junio, por el que se establecen las bases generales del régimen de conciertos entre las universidades y las instituciones sanitarias y se anulan algunos de sus contenidos ${ }^{4}$, la dificultad de encajar todo ese conjunto de tareas en una jornada laboral única ha sido una constante a lo largo del tiempo. Las «plazas vinculadas» han sido tildadas de figura jurídica compleja, conflictiva y hasta contradictoria ${ }^{5}$, con falta de definición y con carencia de un estatuto jurídico actualizado, cosa que por otro lado, ocurre también con el profesorado universitario en general ${ }^{6}$.

Muchos de los titulares de plazas vinculadas comparten un sentimiento de falta de reconocimiento y de injusticia en la consideración de su actividad, frente a unas administraciones, las universidades y las instituciones sanitarias, que consideran que su encaje con el resto de profesionales en uno y otro ámbito produce tensiones. De ahí que en los últimos tiempos se haya planteado la necesidad de una nueva regulación de la figura o incluso la propia continuidad de la misma, cuestión, sin embargo, no exenta de dificultades, por las peculiaridades que revisten los dos ámbitos implicados ${ }^{7}$. El simple hecho de que dos ministerios tengan que buscar una solución conjunta, satisfaciendo las necesidades académicas y las asistenciales, no es cuestión menor ${ }^{8}$. El dato de que el legislador insista a través de distintas leyes en que se precisa un nuevo marco jurídico, sin que se haya llegado a concretar, solo es prueba de lo que se señala.

\section{RJ 9894.}

4 La base décimo tercera del art. 4 se anula precisamente por no incluir la labor investigadora en la dedicación de los profesores vinculados.

5 F. Gurrea Casamayor (1999), «Universidades y Hospitales: régimen jurídico de las plazas vinculadas y de los profesores asociados médicos. Aspectos Docentes y Asistenciales», en IV Curso de régimen de las universidades públicas, Madrid: Lex Nova, págs. 355-440.

6 Baste señalar el RD 898/1985, de 30 de abril, de régimen del profesorado universitario. 7 J. Vázquez Garranzo (2006), «Disposiciones Adicionales», en A. Palomar Olmeda y V. Sempere Navarro (dirs.), Comentarios a la Ley 55/2003, del Estatuto Marco del personal Estatutario de los Servicios de Salud, Cizur Menor: Thomson-Aranzadi, págs. 755-756.

8 El art. 12 de la Ley 44/2003 exige la colaboración permanente entre los organismos de las administraciones públicas competentes en materia de educación y de sanidad, la concertación de las universidades y las instituciones y centros sanitarios y la disposición de toda la estructura del sistema sanitario para ser utilizada en la docencia. A. Perelló Jorquera (2013), «El marco jurídico de las profesiones sanitarias en la LOPS. Régimen de las titulaciones sanitarias. La formación de los profesionales sanitarios», en A. Palomar Olmeda y J. Cantero Martínez (dir.), Tratado de Derecho Sanitario, vol I, Cizur Menor: Thomson Reuters, págs. 1009-1031. 
Además, la viabilidad de este modelo docente se ha cuestionado por varios motivos que han coincidido en el tiempo. El primero de ellos viene dado por una necesidad acuciante de incorporar profesores en el área de Ciencias de la Salud. El envejecimiento de la plantilla se ha hecho notar de forma especialmente relevante en este ámbito y el número de jubilaciones producidas y concentradas en un período de tiempo reducido no se ha podido cubrir con nuevos profesionales docentes.

La crisis económica y la congelación de la tasa de reposición en las Leyes Generales de Presupuestos a partir de 2011 ha afectado de forma perniciosa a las facultades de Medicina; si bien es cierto que, en toda la universidad, la tasa de reposición ha producido unos efectos indeseables y ha repercutido en la calidad de la enseñanza y en el relevo generacional, en la rama de conocimiento de Ciencias de la Salud, las universidades se encuentran con un verdadero problema a la hora de afrontar el relevo generacional. En el mismo período ha coincidido la jubilación de toda una generación de profesionales con la imposibilidad de convocar plazas por parte de las universidades, junto a unos recortes presupuestarios de una intensidad no conocida hasta este momento.

Es cierto que la Ley de Presupuestos Generales del Estado para 2016 ha normalizado la tasa de reposición en el empleo público y que, sin duda, esta medida ayudará a atenuar el problema, pero no podemos olvidar que existe una demanda acumulada durante cinco años, a la que no se va a poder dar solución de un día para otro, salvo que se opte por un arreglo ad hoc.

Las universidades, para afrontar la solución, deben contar además con presupuestos holgados que permitan llevar a cabo las convocatorias. No basta que se puedan convocar nuevas plazas, se debe tener la disponibilidad presupuestaria para asumir el mayor coste de la plantilla docente. En el seno de las instituciones académicas existe otro problema que viene dado porque sea cual sea el número de plazas que se puedan convocar, la tendencia al reparto entre las distintas áreas de conocimiento al que tienden los rectores en aras del «logro de la paz social», impide la acumulación de las convocatorias de plazas en Ciencias de la Salud, no porque exista legalmente ninguna cortapisa, sino porque el resto de ramas del conocimiento se sentirían agredidas, por mucho que se pueda argumentar que las necesidades en este ámbito son más imperiosas que en el resto. Todos los rectores han puesto en evidencia el problema en los últimos ańos, pero dudo mucho que sean capaces de adoptar decisiones contundentes junto a las instituciones sanitarias, a la hora de decidir el reparto de plazas en las convocatorias.

Hay otro elemento que afecta a las convocatorias de las plazas docentes en las áreas de Ciencias de la Salud, y no es otro que la necesidad de contar con profesores acreditados a los cuerpos docentes en estas áreas, o concretándolo más en las específicas áreas donde se produzcan las necesidades docentes. 
El sistema de habilitación diseñado en la Ley Orgánica 6/2001, de 21 de diciembre, de universidades, en un primer momento y de acreditación después (tras la reforma por la LO 4/99, de 7 de abril) puso el acento en la investigación, a la hora de valorar los méritos de los futuros acreditados, lo cual supuso una dificultad añadida para los profesores que en su jornada tienen que atender la labor asistencial y la docente. El peso de las publicaciones, la participación en proyectos de investigación en el ámbito nacional o internacional con financiación obtenida en procedimientos de concurrencia competitiva, las estancias en centros de investigación en el extranjero, son méritos difíciles de lograr para los profesionales sanitarios, cuya trayectoria no es coincidente con la del profesor universitario.

Mientras que el profesor universitario se forma mientras realiza su tesis doctoral tras sus estudios de grado y máster, el graduado en Medicina, tras obtener plaza de MIR, debe formarse como facultativo especialista e intentar su acceso al sistema sanitario, sin perjuicio, de que algunos logren compaginar esa dedicación con la realización de la tesis doctoral. A partir de ese momento, puede estar en mejores condiciones de afrontar el doctorado, pero sigue siendo complejo lograr la redacción de publicaciones con impacto, la presentación de comunicaciones a congresos y, en definitiva, la configuración de una trayectoria con méritos suficientes para intentar la acreditación. Todo ello ha traído consigo que el número de acreditados en las áreas de Ciencias de la Salud sea realmente bajo en relación con el resto de ramas del conocimiento y que para muchos facultativos haya dejado de ser atractiva la carrera académica. Esta cuestión tal vez pueda modificarse en un período razonable de tiempo, si el nuevo sistema de acreditación que acaba de ponerse en marcha facilita la acreditación de los facultativos, sobre todo para los profesores titulares, pero, tras las críticas producidas, tampoco parece que los cambios puedan ser relevantes.

A los factores expuestos hay que añadir el aumento de la oferta de plazas para los estudios de Medicina que se lleva produciendo progresivamente desde 2007. Ante la creciente demanda por parte de los estudiantes, que hace que cada año queden miles de estudiantes fuera de estos estudios, con la frustración social que genera tanto en ellos como en sus familias, y que las notas de acceso sean las más altas de toda la oferta de titulaciones, se han aumentado el número de plazas ofertadas en las facultades. La situación generada se resume en el hecho de que una plantilla mermada de plazas vinculadas se ve en la tesitura de atender a un número considerablemente mayor de estudiantes.

Esta coyuntura ha llevado a que en los últimos años las universidades hayan volcado el peso de la docencia en los profesores asociados de Ciencias de la Salud, única figura que, conforme a la legislación vigente, permite una incorporación inmediata para la cobertura de las necesidades docentes. Sin 
embargo, esta figura no da estabilidad al profesorado, cuenta con una retribución casi simbólica y no permite el desarrollo de una carrera docente e investigadora en condiciones óptimas. Se puede considerar como una figura de acceso, pero no la figura que dé cobertura a los profesionales llamados a ser la fuerza tractora en materia de investigación biomédica y en la formación clínica de los futuros profesionales.

Para eso hacen falta categorías de profesorado estables. Sin una perspectiva de estabilización a corto plazo, esta figura no resulta atractiva para incorporar facultativos a la docencia, la cual se puede resentir en cuanto a su calidad. Sin embargo, sigue siendo la única posibilidad existente y viable en este momento conforme a la normativa vigente, y por la que siguen apostando tanto las universidades como las entidades sanitarias. Por último, aunque carezcan prácticamente de reconocimiento, los numerosos tutores clínicos que apoyan la docencia en los hospitales también contribuyen a sostener al modelo y deben ser tenidos en consideración.

Todos estos factores han planteado la necesidad de modificar el régimen jurídico de las plazas vinculadas, pero sin que de momento se haya producido una propuesta clara al respecto, propuesta que, cómo veremos, no está exenta de dificultades desde el punto de vista jurídico ni organizativo. El modelo que ha cubierto la docencia en Medicina en los últimos treinta años parece estar agotado, por lo que se precisa un debate sobre la cuestión, sin prisa pero sin calma, que proporcione nuevas alternativas o que, simplemente, mejore el actual, sin olvidar, que este modelo ha dado lugar a una formación de máxima calidad reconocida en toda Europa.

\section{RECORRIDO POR LA NORMATIVA REGULADORA}

El origen remoto de este marco normativo lo podemos situar en la Ley de Coordinación Hospitalaria de 21 de julio de 1962, ley que tenía entre sus objetivos coordinar la situación hospitalaria del país y que preveía que los llamados hospitales clínicos se vinculasen con las universidades a través de convenios con el entonces Instituto Nacional de Previsión. La DA 20a de la Ley 50/84, de 30 de diciembre, de Presupuestos, traspasó los hospitales clínicos de algunas universidades del Ministerio de Educación al Ministerio de Sanidad, lo que supuso que los hospitales hasta entonces adscritos a las universidades quedaron fuera de su dependencia demanial, trasladándose esta al INSALUD o a las CC. AA. que ya tuviesen las competencias de sanidad adquiridas? ${ }^{9}$. Esto

$9 \quad$ F. Gurrea Casamayor (1999: 370-372). 
precipitó el diseño de un nuevo marco jurídico que propiciase el encaje de las plantillas docentes universitarias en los órganos de gestión hospitalaria.

El RD 1558/86, de 28 de junio, por el que se establecen las bases generales del régimen de conciertos entre las universidades y las instituciones sanitarias, va a ser el encargado de dar respuesta a esta necesidad, regulando las bases generales del régimen de conciertos entre las universidades y las instituciones sanitarias, normativa que mantiene su vigencia en la actualidad, constituyendo desde ese momento el principal instrumento regulador de las denominadas plazas vinculadas. A pesar del tiempo transcurrido y con algunas modificaciones sufridas, dicho Real Decreto sigue siendo la pieza clave, lo cual no deja de resultar llamativo en un mundo de legislación motorizada, como acostumbramos a decir los juristas. La cuestión sobre la que hay que reflexionar es si esta norma mantiene su vigencia por las bondades de su regulación o por la incapacidad de los $\mathrm{Mi}$ nisterios de Educación y de Sanidad para abordar una tarea que, desde luego, es compleja, pero no por ello debe seguir siento pospuesta.

Cuando se aprobó el RD 1558/86 acababa de entrar en vigor la Ley 14/86, de 25 de abril, General de Sanidad, la cual dedicaba su título VI a la Docencia en el Sistema Nacional de Salud, con dos artículos, el 104 y 105, que introducían novedades importantes en este ámbito. Por un lado, toda la estructura asistencial del sistema sanitario pasa a estar en disposición de ser utilizada para la docencia pregraduada, posgraduada y continuada de los profesionales; por otro, fija el binomio universidades/instituciones sanitarias y señala la competencia de Educación y Sanidad para fijar el régimen de los conciertos, a efectos de garantizar la docencia práctica de la Medicina y Enfermería y otras enseñanzas que así lo requieran. Entre sus exigencias se señala la necesidad de que las universidades cuenten, al menos, con un hospital y tres centros de atención primaria universitarios ${ }^{10}$, o con función universitaria para el ejercicio de la docencia y la investigación concertados. En dichos centros sanitarios «con funciones universitarias» se deberá programar la docencia y la investigación, de manera coordinada por las autoridades universitarias y sanitarias, en el marco de sus competencias, para lo que se contempla la necesaria participación de las universidades en sus órganos de gobierno.

El art. 105, que no había sido previsto en la redacción inicial de la ley, va a ser, sin embargo, el que realmente cree y defina las llamadas plazas vinculadas. Señalaba este precepto en su redacción originaria que, en el marco de la

10 El concepto de centro de atención primaria universitario queda diluido en las áreas sanitarias que se fijan con posterioridad. En este sentido, A. López de la Osa Escribano (2009), «Universidad y Sanidad: especial referencia a los estudios de Medicina», en J. González García (dir.), Comentarios a la Ley Orgánica de Universidades, Madrid: Civitas, págs. 803 a 829 . 
planificación asistencial y docente de las administraciones públicas, el régimen de concierto entre las universidades y las instituciones sanitarias podría establecer la vinculación de determinadas plazas asistenciales de la institución sanitaria con plazas docentes de los cuerpos de profesores de universidad. Estas plazas así vinculadas se proveerían a través de un concurso, en el que podrían participar los candidatos que reuniesen los requisitos señalados en la LRU (Ley Orgánica 11/1983, de Reforma Universitaria), siempre que acreditasen además estar en posesión del título de especialista. También se regulaban las especialidades de los concursos de acceso, en cuanto a las comisiones encargadas de resolver y las pruebas a realizar. Junto a las plazas vinculadas, se preveía que los conciertos pudiesen establecer asimismo un número de plazas de profesores asociados que debería cubrirse por personal asistencial que estuviese prestando servicios en la institución sanitaria concertada, y también la posibilidad de reconocer plazas de ayudantes en las plantillas de las universidades ${ }^{11}$.

11 Este precepto ha sido modificado por la disposición final segunda de la Ley Orgánica 4/07, de 12 de abril, adaptando su contenido a las figuras de profesorado que contempla la LOU.

«1. En el marco de la planificación asistencial y docente de las administraciones públicas, el régimen de conciertos entre las universidades y las instituciones sanitarias podrá establecer la vinculación de determinadas plazas asistenciales de la institución sanitaria con plazas docentes de los cuerpos de profesores de universidad y con plazas de profesor contratado doctor.

"Las plazas así vinculadas se proveerán por concurso entre quienes hayan sido seleccionados en los concursos de acceso a los cuerpos de funcionarios docentes universitarios o a plazas de profesor contratado doctor, conforme a las normas que les son propias. "Quienes participen en los procesos de acreditación nacional, previos a los mencionados concursos, además de reunir los requisitos exigidos en las indicadas normas, acreditarán estar en posesión del título de Especialista en Ciencias de la Salud que proceda y cumplir las exigencias que, en cuanto a su cualificación asistencial, se determinen reglamentariamente. Asimismo, las comisiones deberán valorar los méritos e historial académico e investigador y los propios de la labor asistencial de los candidatos y candidatas, en la forma que reglamentariamente se establezca.

"En las comisiones que resuelvan los mencionados concursos de acceso, dos de sus miembros serán elegidos por sorteo público por la institución sanitaria correspondiente.

"2. Los conciertos podrán establecer, asimismo, un número de plazas de profesores asociados que deberá cubrirse por personal asistencial que esté prestando servicios en la institución sanitaria concertada. Este número no será tenido en cuenta a los efectos del porcentaje de contratados que rige para las universidades públicas. Estos profesores asociados se regirán por las normas propias de los profesores asociados de la universidad, con las peculiaridades que reglamentariamente se establezcan en cuanto 
El RD 1558/86 trató de garantizar que los conciertos asegurasen la calidad docente en la formación de los futuros médicos, exigiendo una serie de requisitos a las universidades que ofertasen la licenciatura en Medicina y Cirugía. Así, estas deberían disponer al menos de un hospital y tres centros de atención primaria de carácter universitario ${ }^{12}$, haciendo coincidir estas instituciones con las de mayor calidad asistencial del ámbito geográfico correspondiente. Esta previsión llama la atención, pues pone en evidencia que el Gobierno en aquel momento no preveía el incremento de universidades (especialmente privadas), y más concretamente el aumento de facultades de Medicina que se iba a producir en nuestro país en los siguientes treinta años ${ }^{13}$.

Los hospitales y centros de atención primaria concertados pasan a constituir las unidades docente-asistenciales de los centros universitarios, proporcionando, en coordinación con la infraestructura sanitaria correspondiente, asistencia del máximo nivel científico y desarrollando funciones de investigación en las áreas de Ciencias de la Salud. Para ello se exige que las universidades, mediante los oportunos conciertos, garanticen que los profesores universitarios pertenecientes a los cuerpos de funcionarios docentes, cuya actividad docente así lo exija, puedan ejercer la complementaria actividad asistencial, en los términos que se fija en el propio Real Decreto y en los respectivos conciertos. Asimismo, estos deberán considerar las necesidades hospitalarias derivadas de la investigación que se realice en las universidades. También se permitió que las universidades pudiesen concertar igualmente con

al régimen temporal de sus contratos. Los estatutos de la universidad deberán recoger fórmulas específicas para regular la participación de estos profesores y profesoras en los órganos de gobierno de la universidad.

"3. Los conciertos establecerán, asimismo, el número de plazas de ayudante, profesor ayudante doctor y profesor contratado doctor en las relaciones de puestos de trabajo de las universidades públicas, que deberán cubrirse mediante concursos públicos entre profesionales sanitarios que hubieran obtenido el título de especialista en los tres años anteriores a la convocatoria del concurso».

12 Igual previsión se mantiene en el RD 420/2015, de 29 de mayo, de creación, reconocimiento, autorización y acreditación de universidades y centros universitarios en el anexo II. Las universidades deberán contar al menos con un hospital y tres centros de atención primaria (de titularidad pública o privada), con base en un concierto en el caso de las universidades públicas, o en un convenio, en el de las universidades privadas.

13 Para el curso 2017-2018 se ofertarán 6995 plazas en las facultades de Medicina españolas, 118 más que en el curso anterior, de las que 1310 corresponden a universidades privadas. De hecho, este año comienza su andadura la universidad de Vic de carácter privado, con una oferta de 80 plazas. 
instituciones sanitarias de titularidad privada, siempre que estén acreditadas y que cumplan los requisitos fijados en la propia normativa ${ }^{14}$.

En cuanto a los conciertos, la base sexta del art. 4 contemplaba la necesidad de que señalasen los servicios de la institución sanitaria que se conciertan y los departamentos universitarios que con ellos se relacionan, así como las fórmulas de coordinación entre las actividades docentes, investigadoras y asistenciales. Los servicios así concertados lo serán en su totalidad y el concierto preverá la existencia de una comisión universidad-institución sanitaria compuesta por un número igual de miembros de ambas, encargada de velar por la correcta aplicación del mismo, teniendo en cuenta, de un lado, la estructura departamental prevista en la legislación universitaria y, de otra, la estructura funcional de la institución sanitaria. Por tanto, los servicios pasan a ser el nivel orgánico de referencia para el concierto.

Siguiendo con el contenido del RD 1558/1986, en el art. 4 también se señala que el concierto establecerá las plazas de facultativos especialistas de la institución sanitaria que quedan vinculadas con plazas docentes de la plantilla de los cuerpos de profesores de la universidad. Mientras tenga tal carácter, dicha plaza se considerará a todos los efectos como un solo puesto de trabajo y supondrá para quien la ocupe el cumplimiento de las funciones docentes y asistenciales

14 Al referirse a universidades, es evidente que está danto el mismo tratamiento a las universidades públicas y a las privadas, cuestión que permitiría incluir a estas últimas, cuando años después se produjese la proliferación de universidades privadas con oferta en estudios de Medicina. El RD 420/2015, de 29 de mayo, de creación, reconocimiento, autorización y acreditación de universidades y centros universitarios, considera que, en lugar de concierto, procederá un convenio de vinculación con la entidad sanitaria en el caso de las universidades privadas (anexo II y disposición final tercera por la que se modifica el RD 1558/1986). La base quinta del RD 1558/1986 pasa a tener la siguiente redacción: «Se utilizará la denominación "hospital universitario" cuando el concierto se refiera al hospital en su conjunto o que abarque la mayoría de sus servicios y/o unidades asistenciales, en el caso de que solo se concierten algunos servicios, se hablará de "hospital asociado a la universidad". Lo mismo se aplicará a los centros de atención primaria.

Un hospital universitario solo podrá estar vinculado por concierto o convenio a una universidad para la impartición de una misma titulación. Excepcionalmente, con la finalidad exclusiva de la realización de prácticas y con base en convenios específicos, podrá haber estudiantes de otras universidades, previa consulta por escrito a la universidad vinculada».

En algunas CC.AA. se ha generado un cierto problema con las universidades privadas, que muchas veces, sin un instrumento jurídico, vienen utilizando la red pública para su docencia clínica. Valencia, en concreto, se está planteando que solamente puedan utilizar los hospitales privados o concertados, pero nunca los públicos. 
en los términos que se contemplan en la propia norma, igualmente establecerá el número de plazas de profesor asociado pertenecientes a la plantilla de la universidad que obligatoriamente deberán cubrirse por personal de la institución sanitaria concertada. Y, aunque a continuación se señala, que «cuando se defina la plantilla vinculada se establecerá la adecuada correspondencia entre la actividad docente y asistencial para hacer efectivas ambas funciones», en el acceso a las jefaturas de departamento, servicio o sección u otra jefatura de las instituciones sanitarias, se excluye la participación de la universidad al señalarse que este acceso «deberá realizarse conforme a las disposiciones por las que las administraciones sanitarias competentes regulen el acceso a las mismas». Si bien algunos conciertos contemplan la comunicación a la comisión de seguimiento en el supuesto de tratarse de plazas vinculadas o vinculables ${ }^{15}$.

Esto tiene como consecuencia, como muy acertadamente se ha apunta$\mathrm{do}^{16}$, y los conciertos celebrados al amparo de este $\mathrm{RD}$ han corroborado, que la vinculación docente asistencial no contempla la equiparación de las diferentes figuras de profesorado existentes en la universidad con las existentes en las instituciones sanitarias, lo que puede llevar a que un catedrático de universidad pueda ocupar una plaza de facultativo especialista en el ámbito asistencial $y$, al revés, que un jefe de servicio en un hospital universitario ocupe plaza de profesor asociado de Ciencias de la Salud.

En realidad, la equiparación que fija el RD 1558/1986 quedaría limitada a la de facultativo especialista de área, pues la provisión de las jefaturas de servicio o de sección, o ahora de director de unidad de gestión clínica, se someterán a las normas existentes en cada momento en el ámbito sanitario. Probablemente, las disfunciones que esto traería consigo no fueron consideradas en su momento y, aunque el paso del tiempo las ha puesto sobre la mesa, la posible solución a las mismas no es fácil por una serie de condicionantes que serán analizados a continuación.

El resultado de todo ello es que se ocupa una única plaza con dedicación compartida que puede estar al máximo nivel en un ámbito y a un nivel básico en el otro. Y esta falta de equivalencia jerárquica, junto con la doble dependencia, ha causado numerosas disfunciones. Es cierto que la disponibilidad de plazas en uno y otro ámbito sigue sus propios criterios y necesidades, pero la situación en la que se encuentran muchos profesionales no deja de ser paradójica, por no decir absurda. Lo que sí contemplan los conciertos con carácter general es que la convocatoria de puestos en el ámbito sanitario (jefes

15 Concierto Gobierno del Principado de Asturias-Universidad de Oviedo, 5 noviembre de 2009.

16 A. López de la Osa Escribano, en «Universidad y Sanidad: especial referencia a los estudios de Medicina», op. cit., pág. 814. 
de servicio, jefes de sección, directores de unidad clínica o directores de área clínica) tenga en cuenta los méritos docentes y de investigación, y, de hecho, formalmente los baremos tienen en cuenta la docencia universitaria y las diferentes categorías que se han ocupado a lo largo del tiempo. No ha ocurrido así en el ámbito académico en los últimos ańos, sino a la inversa, dada la poca relevancia que a la hora de valorar los méritos para las acreditaciones docentes ha tenido la labor asistencial, incumpliendo, por cierto, una exigencia que está en el art. 105 de la LGS.

Se han suscitado casos, y así se ha pormenorizado en algún trabajo, que han generado dudas en las propias comisiones mixtas de seguimiento de los conciertos que han ido buscando soluciones para cada supuesto concreto ${ }^{17}$. Así, puede ocurrir que un profesor titular con plaza vinculada pueda acceder a una cátedra que no se convoque como vinculada, lo cual le generaría automáticamente una situación de incompatibilidad entre la pertenencia al nuevo cuerpo funcionarial y la plaza asistencial que venía ocupando en el ámbito sanitario ${ }^{18}$. También podría ocurrir que la plaza de catedrático se convocase con carácter vinculado a un puesto de facultativo especialista y que ese profesor titular tuviese previamente en el ámbito asistencial un puesto de jefe de servicio, en ese caso, y salvo que se estableciese en las bases de la convocatoria que se le respetaría el puesto de trabajo asistencial previo, la obtención de la cátedra supondría la degradación en el ámbito asistencial. La vinculación al puesto de jefe de servicio no sería posible porque dicha plaza ya estaría ocupada precisamente por el profesor titular con plaza vinculada y puede ocurrir que quien obtenga la cátedra convocada no sea el primer profesor titular, sino un profesor titular procedente de otra universidad. Las posibilidades son variadas, y los resultados, a veces, absurdos. También es cierto que cuando se reúne la categoría de jefe de servicio y se accede a una cátedra, con frecuencia se prevé en la convocatoria el reconocimiento del puesto de trabajo previo que se viniese ocupando en el ámbito asistencial, como forma de atenuar las disfuncionalidades. Igualmente, la aprobación de nuevos conciertos suele incluir cláusulas en las que se señala que se respetará la equivalencia docente asistencial vigente en el momento de entrada en vigor del concierto ${ }^{19}$.

17 F. Gurrea Casamayor (1999: 423 y ss.).

18 La única previsión que hace el RD 1558/86 en relación a un posible cambio de categoría en el ámbito académico se refiere al supuesto de que un profesor asociado saque una plaza de profesor titular, que no esté previamente vinculada, ya que, en ese caso, se vinculará por la Comisión, siempre que las necesidades asistenciales y docentes así lo aconsejen, y se amortizará el correspondiente contrato (base novena; apdo. 2).

19 Concierto Gobierno del Principado de Asturias-Universidad de Oviedo, 5 noviembre de 2009. 
Algunas sentencias han resuelto esta cuestión, así la del TSJ de Madrid, de 22 de noviembre de $2003^{20}$, donde se apunta el carácter discrecional del nombramiento y cese de los jefes de servicio, señalando que la correspondencia entre catedrático y jefe de sección o jefe de servicio no es automática, estando condicionado el acceso a dichas jefaturas al cumplimiento de las disposiciones específicas que lo regulen en cada momento. Así, una plaza de catedrático puede vincularse a una de facultativo especialista, que con el transcurso del tiempo puede ser nombrado jefe de servicio y, tras un tiempo, ser cesado, teniendo la Administración Sanitaria plena competencia para acordar dicho cese «sin que fuera necesaria la concurrencia en el mismo sentido de la universidad, pues el referido cese en modo alguno suponía modificar unilateralmente el contenido de una plaza vinculada por la sencilla razón de que tal vinculación solo existía en relación a la plaza de facultativo especialista» .

\section{ASPECTOS JURÍDICOS A TENER EN CUENTA ANTE UNA NUEVA REGULACIÓN DE LAS PLAZAS VINCULADAS}

\section{DIFERENCIAS EN EL ACCESO A LAS PLAZAS DE CUERPOS DOCENTES UNIVERSITARIOS Y A LOS PUESTOS DIRECTIVOS ASISTENCIALES}

En estos momentos, los cuerpos docentes universitarios son dos, conforme a la Ley Orgánica 6/2001, de 21 de diciembre, de Universidades (LOU): el cuerpo de catedráticos de universidad y el cuerpo de profesores titulares de universidad (art. 56). Ambos cuerpos tienen plena capacidad docente e investigadora y sus funciones en la universidad son esencialmente las mismas, salvo la necesidad de ser catedrático de universidad para ocupar el puesto de rector (art. 20.2 LOU) y el imperativo de que las comisiones de acreditación o las que juzgan los concursos a cátedra deban estar integradas en su totalidad por catedráticos (arts. 4-6 del RD 1312/2007 de 5 de octubre y art. 6 RD $1313 / 2007$, de 5 de octubre, por el que se regulan los concursos de acceso a cuerpos docentes).

Constituye esta realidad del ámbito docente universitario una excepción y peculiaridad, pues existen dos cuerpos funcionariales a los que se exige para el acceso, en todo caso, estar en posesión del título de doctor y pertenecen los dos al grupo A1, lo que está llevando a que cada vez se perfile el acceso al cuerpo de catedráticos más como una promoción profesional dentro de

20 Sala de lo Contencioso-Administrativo, Sección 7a, Sentencia 1089/2003. 
la carrera académica ${ }^{21}$. Sin embargo, no se puede obviar que el cambio de categoría supone un cambio de cuerpo funcionarial que obliga a superar una nueva acreditación y un nuevo concurso; no se trata de ocupar un puesto de más responsabilidad manteniendo la misma condición funcionarial, sino que esa condición funcionarial se altera. Tampoco se trata de una promoción interna, ya que la promoción interna, como es bien sabido, supone el cambio de un grupo inferior a uno superior y aquí ambos cuerpos pertenecen al grupo A1 (art. 16 del Estatuto Básico del Empleado Público [TR aprobado por el Decreto Legislativo 5/2015, de 30 octubre]).

En relación con las plazas vinculadas, la LOU no aporta novedades importantes ${ }^{22}$, simplemente en el art. 61 recuerda que el personal de los cuerpos de funcionarios docentes universitarios que ocupen una plaza vinculada a los servicios asistenciales de instituciones sanitarias, en áreas de conocimiento de carácter clínico asistencial, de acuerdo con lo establecido en el art. 105 de la LGS, se regirá por los preceptos de la propia ley que le sean de aplicación.

Sí es importante el hecho de que eleva a rango legal lo ya previsto en el RD 1558/1986: la consideración de las plazas vinculadas, a todos los efectos, como un solo puesto de trabajo, lo cual implica que cualquier alteración en relación con esta configuración exigiría la modificación de la LOU. Aunque la LOU parece adjudicarse una prevalencia en la regulación ${ }^{23}$, lo cierto es que la aplicación de la normativa universitaria queda modulada por los contenidos de la LGS y, sobre todo, por la remisión que hace a una futura regulación conjunta, ya que prevé que el Gobierno, a propuesta conjunta de los ministros de Educación, Cultura y Deporte y de Sanidad y Consumo y, en su caso, de Defensa (en relación con los hospitales militares), establezca normativa de desarrollo en relación con estos funcionarios y, en particular, se señala que en estas normas se determinará el ejercicio de las competencias sobre situaciones administrativas, se concretará el régimen disciplinario de este personal y se establecerá, a propuesta del Ministro de Hacienda, a iniciativa conjunta de los ministros indicados en el inciso anterior, el sistema de retribuciones aplicable al mencionado personal. Este desarrollo normativo no se ha producido hasta la fecha, y la implicación

21 Paradójicamente, desde el Decreto Ley 14/2012, de 20 de abril, un profesor titular con tres sexenios impartirá 16 créditos, mientras que al catedrático le corresponderán 24 créditos en la misma situación. Lo cual implica que un titular que logre acceder al cuerpo de catedráticos con tres sexenios asumirá mayores obligaciones docentes que cuando era profesor titular (art. 68 LOU).

22 J. M. Souvirón Morenilla y F. Palencia Herrejón (2002), La nueva regulación de las universidades. Comentarios y análisis sistemático de la LO 6/2001, de 21 de diciembre, de universidades, Granada: Comares, págs. 493-497.

Ibid., pág. 496. 
de al menos dos Ministerios en el asunto hace indudablemente más difícil la iniciativa $^{24}$.

Desde el año 2007, el acceso a los cuerpos de funcionarios docentes universitarios exige la previa obtención de una acreditación nacional, acreditación que tiene como objetivo la calidad en la selección del profesorado funcionario (art. 57 LOU). Precisamente este sistema de acreditación no ha tenido efectos positivos en el ámbito de Ciencias de la Salud, como se ha apuntado en la introducción. La existencia de una única comisión de acreditación en esta rama de conocimiento, que evaluaba al mismo tiempo perfiles investigadores y perfiles asistenciales, hizo difícil la acreditación de aquellos docentes con un perfil predominantemente asistencial. El poco peso de la actividad profesional en el baremo, ya que esta solo constituía un apartado residual dentro de la evaluación de actividad docente, tanto en las acreditaciones a profesor titular como en las de acceso a cátedras, dificultó enormemente la obtención de la misma.

El procedimiento y los méritos se encuentran regulados en el ya mencionado RD 1313/2007 de 5 de octubre, modificado por el RD 415/2015, de 29 de mayo, en el cual se configura un nuevo sistema de acreditación que apuesta por el acercamiento de las comisiones a la realidad de las diferentes áreas de conocimiento. En el área de Ciencias de la Salud, el cambio implica la creación de tres comisiones de acreditación: una para Ciencias Biómédicas ${ }^{25}$, otra de Medicina Clínica y Especialidades Clínicas ${ }^{26}$, y otra de Especialidades Sanitarias ${ }^{27}$. Ciertamente, esto puede ser un paso para que los criterios de evaluación se aproximen más a la realidad de cada una de las especialidades. Además, entre los méritos evaluables, que se organizan ahora en cinco bloques de actividad, junto a la formación académica, la docencia, la investigación y la gestión, se incluye la transferencia de conocimiento y experiencia profesional, en la que, a su vez, se incorpora un apartado dedicado a la calidad y dedicación a

24 En el año 2001, la LOU recogió esta necesidad. Dos años después, la Ley 44/2003, de 21 de noviembre, de ordenación de las profesiones sanitarias, en su art. 14, se refiere a los conciertos entre universidades y administraciones sanitarias, reiterando la necesidad de una nueva normativa.

25 Anatomía Patológica. Anatomía y Anatomía Patológica Comparadas. Anatomía y Embriología Humana. Fisiología. Histología. Inmunología. Microbiología. Parasitología. Toxicología.

26 Cirugía. Dermatología. Estomatología. Medicina. Medicina Legal y Forense. Obstetricia y Ginecología. Oftalmología. Otorrinolaringología. Pediatría. Psiquiatría. Radiología y Medicina Física. Urología. Traumatología y Ortopedia.

27 Enfermería. Fisioterapia. Farmacia y Tecnología Farmacéutica. Farmacología. Nutrición y Bromatología. Medicina Preventiva y Salud Pública. Medicina y Cirugía Animal. Sanidad Animal. 
actividades profesionales en empresas, instituciones, organismos públicos de investigación u hospitales, distintas a las docentes o investigadoras.

La nueva evaluación permite entre las varias combinaciones posibles que se establecen en el Real Decreto $415 / 15$ en su anexo II, que con una evaluación $\mathrm{A}$ en la experiencia profesional, una $\mathrm{B}$ en docencia y una $\mathrm{C}$ en investigación se pueda lograr la acreditación, tanto al cuerpo de catedráticos como al cuerpo de profesores titulares. Tras casi un año de paralización de los procedimientos de acreditación, se han vuelto a iniciar los procedimientos de acreditación ANECA, por lo que, en poco tiempo, podremos valorar si el nuevo baremo permite un mayor número de acreditados en las áreas clínicas de Ciencias de la Salud ${ }^{28}$.

Una vez que se obtiene la acreditación, la carrera de obstáculos continúa, y es necesario que exista una convocatoria de plazas en una universidad y que los acreditados se presenten y superen el concurso. En la composición de las comisiones que han resolver los concursos de acceso, el RD 1313/2007, en su art. 6.5, recoge la presencia de dos miembros que ostenten el título de especialista que se exija como requisito para concursar a la plaza, que serán elegidos por sorteo público por la institución sanitaria correspondiente, entre el correspondiente censo público que anualmente comunicará al Consejo de Universidades. Estos miembros tendrán que ser doctores como el resto de miembros de la comisión.

Además, es preciso tener en cuenta lo previsto en el art. 105 de la LGS, que requiere que las plazas vinculadas se ocupen por quienes previamente tenga el título de especialistas en Ciencias de la Salud y presten sus servicios en el centro concertado o centros concertados. Igual requisito se fija para la provisión de plazas de profesores ayudantes, ayudantes doctores y contratados doctores (solamente esta última categoría tiene carácter estable), si bien en estos supuestos se exige que la titulación se haya obtenido en los tres últimos años.

En relación con la incorporación de estas figuras contractuales para cubrir las necesidades de la docencia clínica, lo cierto es que las mismas no se

28 Con el antiguo sistema, es cierto que tanto el número de solicitudes como el de acreditaciones era más bajo que en otras ramas del conocimiento. El último informe publicado por ANECA recoge que se solicitaron en 2014340 solicitudes para el cuerpo de profesor titular, de las cuales fueron acreditadas el $46 \%$ y, en el caso del cuerpo de catedráticos, se solicitaron 191, con un éxito del 77\%. En cuanto a las cátedras, el número de solicitudes fue el más bajo en relación con otras ramas de conocimiento, pero el porcentaje de éxito fue el segundo más alto. En cambio, en relación con las titularidades, el número de solicitudes fue el tercero más alto y el porcentaje de éxito fue claramente el más bajo. 
ajustan bien al modelo de plazas vinculadas existentes hasta la fecha y no resultan especialmente atractivas para los profesionales sanitarios, a pesar de que lograr la acreditación requiera menos méritos. Para las plazas de profesores asociados de Ciencias de la Salud no se requiere la titulación de especialista, pero sí que se venga prestando labor asistencial en la institución concertada, de forma que, si por alguna circunstancia causase baja en la plaza asistencial, cesará en la plaza docente.

Hasta aquí el acceso desde el punto de vista docente, sin embargo este no es para nada coincidente con el acceso a las plazas y puestos en el ámbito asistencial. Los facultativos especialistas, una vez que obtienen su plaza como personal estatutario sanitario, pueden optar a distintos puestos a lo largo de su carrera profesional, entre ellos las jefaturas de sección o de servicio, o las diferentes direcciones de unidad de gestión clínica. El hecho de que ocupen uno de estos puestos no altera su clasificación profesional desde el punto de vista estatutario, que seguiría siendo la misma.

$\mathrm{El}$ acceso a los puestos directivos en las instituciones sanitarias ha sufrido diversas regulaciones desde la aprobación del RD 1558/86, primero en el ámbito estatal y más tarde en el autonómico. Sin hacer un recorrido exhaustivo, el RD 118/91, de 25 de enero, otorgó una clara prevalencia al sistema de libre designación en la provisión, lo que implicaba a su vez la posibilidad de libre cese. Esta norma recogía de forma expresa en su DF primera la posibilidad de que los titulares de las plazas vinculadas pudiesen acceder a los puestos de carácter directivo y de jefatura de unidad en las distintas instituciones sanitarias.

El RD fue derogado por el Real Decreto Ley $1 / 99$, de 8 de enero ${ }^{29}$, que dio lugar meses después a la Ley 30/99, de 5 de octubre, que reguló los procedimientos de selección y provisión de plazas de personal estatutario. En su disposición adicional undécima hacía referencia a la provisión de puestos de jefe de servicio y de sección de carácter asistencial en el Instituto Nacional de la Salud. Esta se haría mediante convocatoria pública, en la que podrían participar todos los facultativos con nombramiento como personal estatutario fijo que ostentasen plaza en las instituciones sanitarias de la Seguridad Social, mediante un proceso de selección basado en la evaluación del currículum profesional de los aspirantes y en un proyecto técnico relacionado con la gestión de la unidad asistencial. Los aspirantes seleccionados obtenían un nombramiento temporal para el puesto, sujeto a evaluaciones cuatrienales a efectos de

29 Tras la STC 203/98, que declaró que el art. 34 apartado cuatro de la Ley 4/90, de 29 de junio, resultaba inconstitucional por su inclusión en una Ley de Presupuestos. Este precepto había modificado los sistemas de selección de personal y de provisión de plazas y puestos de trabajo en las instituciones sanitarias de la Seguridad Social y el RD 118/91 lo desarrollaba, viéndose afectada la propia validez del mismo. 
su continuidad. Esta regulación es importante porque ha servido de referente a las regulaciones autonómicas que se fueron aprobando con posterioridad y que desplazaron su aplicación ${ }^{30}$.

Por su parte, la Ley 55/2003, de 16 de diciembre, del Estatuto Marco del personal estatutario de los servicios de salud ${ }^{31}$, en cuanto a la provisión de puestos de trabajo, reitera la necesidad de que esta se ajuste a los principios de mérito y capacidad, dejando en manos de cada servicio de salud la decisión sobre los puestos que han de ser provistos por libre designación (art. 29.3) ${ }^{32}$, mientras que la selección de personal estatutario fijo se hará con carácter general a través del sistema de concurso-oposición, aunque también cabe el sistema de oposición (art. 30). Respecto a las plazas vinculadas, la DA novena señala que se proveerán por los sistemas establecidos en las normas específicas que resulten aplicables, sin perjuicio de que sus titulares queden incluidos en el ámbito de aplicación de esta ley en lo relativo a su prestación de servicios en los centros sanitarios.

30 A modo de ejemplo, la Ley 4/2006, de 22 de diciembre, de medidas sociales y económicas, de la Comunidad de Madrid, deja sin efecto, en el ámbito de la Comunidad de Madrid, los artículos 16.1, 29 , 30 , 31, 32, 33 y 34 del Real Decreto Ley 1/1999, de 8 de enero, sobre Selección de personal estatutario y provisión de plazas en las instituciones sanitarias de la Seguridad Social.

31 A partir de su entrada en vigor, se considera con carácter general que las cuestiones sobre el personal estatutario, incluidos los profesores vinculados, pasan a ser competencia de la jurisdicción contencioso-administrativa y no de la jurisdicción social. Así lo estableció, tras bastantes dudas al respecto, el Auto de la Sala de Conflictos del Tribunal Supremo de 20 de junio de 2005 (RJ 4466).

32 Una crítica clara al sistema de libre designación en la provisión de jefaturas de servicio frente al modelo anterior de concurso-oposición la podemos encontrar en R. Vela Navarrete et al. (2008), «La figura del jefe de servicio: ¿Qué perfil debe tener?», Actas Urológicas españolas, págs. 673-679. Con carácter general, el estatuto marco otorga a la libre provisión carácter excepcional, vinculándola a la selección de puestos de alta responsabilidad: J. A. Monterrubio Quirós (2006), «Provisión de plazas, selección y promoción interna», en A. Palomar Olmeda y V. Sempere Navarro (dirs.), Comentarios a la Ley 55/2003, del Estatuto Marco del personal Estatutario de los Servicios de Salud, Thomson-Aranzadi, págs. 278-279. V. Lomas Hernández (2017), «Provisión de plazas de personal estatutario de los Servicios de Salud», en V. Lomas Hernández (coord.), Régimen Jurídico del Personal Estatutario de los Servicios de Salud, Thomson Reuters, págs. 389 y ss. La jurisprudencia también ha considerado que se deben justificar las razones para que una categoría determinada sea provista por el sistema de libre designación, y que no es adecuado que un decreto establezca con carácter general la provisión de todos los puestos por este sistema, STS 21 de mayo de 2012 (RJ 6908). En el mismo sentido, STSJ de Asturias, de 26 de septiembre de 2014, n 90176/2014). 
En el ámbito autonómico, en los últimos años se han ido aprobando nuevas Leyes reguladoras del personal estatutario, aunque también es cierto que, en otras, las convocatorias de puestos directivos o de responsabilidad se siguen rigiendo por la anterior normativa estatal. Solo a modo de ejemplo, y por ser relativamente recientes, podemos hacer referencia a la Ley 2/2007, de 7 de marzo, del estatuto jurídico del personal estatutario del servicio de salud de Castilla y León, y a la Ley 9/2010, de 23 de diciembre, de personal estatutario de instituciones sanitarias de Cantabria. La Ley de Castilla y León establece el procedimiento de libre designación para la provisión de los puestos de trabajo de carácter directivo y los puestos de trabajo de jefe de servicio y jefe de unidad, lo cual implica también la libre remoción en cualquier momento (art. 38). Con carácter general, el nombramiento y permanencia en el puesto será por cuatro años, siendo luego objeto de nueva evaluación y pudiendo presentarse a la nueva convocatoria quienes lo ocupaban mediante el sistema de libre designación, una vez transcurrido el período de cuatro años. En los casos de supresión del puesto desempeñado mediante el sistema de libre designación se procederá al cese del personal nombrado en el mismo. Por su parte, la Ley 9/2010 de Cantabria señala también la libre designación como procedimiento normal de provisión de los puestos directivos y de las jefaturas de unidad (arts. 49-50) y para las jefaturas de servicio y jefaturas de sección articula un proceso similar al regulado en su día para el INSALUD (art. 52).

Tras este recorrido, se puede afirmar que, mientras que el acceso a una plaza de profesor titular o catedrático es definitiva y estable, haciéndose su provisión mediante el correspondiente concurso, el acceso a un puesto de responsabilidad en el ámbito asistencial puede tener carácter temporal (aunque luego pueda prolongarse $y$, de hecho, muchos jefes de servicio lleven años en sus puestos), bien porque se provea por el sistema de libre designación, bien porque el acceso se condicione a una evaluación transcurrido un determinado período de tiempo que, en el caso de resultar negativa, implicaría la pérdida del puesto.

Por ello, una primera cuestión a resolver para avanzar hacia la equivalencia jerárquica en los dos ámbitos sería atenuar las diferencias en los sistemas de provisión, lo cual afectaría ineludiblemente al ámbito asistencial. En este ámbito, la plaza de facultativo especialista es la que tiene carácter permanente y por eso en su día se optó por la vinculación a estas plazas y no a puestos o categorías concretas, por lo que la solución de esta cuestión pasaría por excepcionar el régimen general de provisión de puestos en el ámbito asistencial para los profesores vinculados, cuestión no exenta de dificultades. 


\section{FALTA DE EQUIVALENCIA ENTRE EL NÚMERO DE PROFESORES Y LOS PUESTOS DE RESPONSABILIDAD EN EL ÁMBITO ASISTENCIAL}

Otra cuestión a tener en cuenta, aunque en los últimos años se ha desdibujado por la falta de convocatorias, viene dada por el hecho de que en la universidad los profesores universitarios no se vinculan stricto sensu a una RPT, a pesar de que el art. 3 del RD 898/1985, de 30 abril, señala que cada universidad establecerá anualmente en el estado de gastos de su presupuesto su plantilla de profesorado, en la que se relacionarán debidamente clasificadas todas las plazas de profesorado.

Las necesidades docentes y el relevo generacional, además de la disponibilidad presupuestaria, son los factores que marcan la convocatoria de plazas. Igualmente, influye el que haya o no profesores acreditados para los distintos cuerpos docentes o para las figuras de contratado doctor o ayudante doctor.

Todo ello no impide que pueda haber en un momento dado en un área de conocimiento varios profesores titulares y varios catedráticos, de hecho, la recuperación de la tasa de reposición al $100 \%$ y la acumulación de profesores acreditados pueden llevar a que se produzca esta situación.

El área de conocimiento con un solo catedrático al frente pertenece al pasado en la mayor parte de los casos, sin embargo, en un ámbito muy jerarquizado como es el hospitalario, resulta difícil imaginar que pueda haber varios jefes de servicio en un área asistencial de un mismo hospital. Ello es difícil de articular y aunque es posible generar puestos de adjuntos o de mandos intermedios, si se hace una equivalencia a un determinado puesto, puede limitar la convocatoria de nuevas plazas vinculadas, aunque las necesidades docentes lo demandasen.

En el ámbito hospitalario sí se cuenta con RPTs y aunque tal vez en el futuro se apueste por una gestión más horizontal ${ }^{33}$, lo cierto es que, a día de hoy, el hospital sigue caracterizándose por el escalonamiento y por la jerarquía de puestos, con lo cual, aunque en un momento perfecto se lograse una plantilla adecuada de profesores vinculados para atender la docencia, difícilmente todos ellos podrían tener un puesto de responsabilidad en el ámbito sanitario. Esta cuestión puede ser solventada en aquellos supuestos en los que existiesen varias entidades concertadas con una única universidad de forma que hubiese varios puestos de responsabilidad con los que se pudieran vincular las plazas.

33 En esta dirección podemos enmarcar el Proyecto de Real Decreto que fija las bases para la implantación de las unidades de gestión clínica en el ámbito de los servicios de salud (si bien, en algunas CC.AA. — como Andalucía o Asturias- vienen funcionando desde hace tiempo). 
Otra cuestión a tener en cuenta y que también crea disfunciones es la falta de correspondencia entre las estructuras universitarias y las de los hospitales. No resulta fácil incardinar los departamentos universitarios de las áreas clínicas en la estructura de los hospitales ${ }^{34}$.

\section{INCREMENTO DE LAS TAREAS DOCENTES CON EL EEES Y SU COMPATIBILIDAD CON LOS PUESTOS DE RESPONSABILIDAD ASISTENCIAL}

La regulación que se contiene en el RD 1558/86 respecto a las funciones docentes es totalmente obsoleta y no se ajusta para nada a la actual situación tras la adaptación al EEES ${ }^{35}$. El RD 1558/86 preveía y prevé que todos los profesores que ocupasen una plaza vinculada desarrollarían el conjunto de sus funciones docentes, investigadoras y asistenciales en una misma jornada — jornada que, con carácter general, en la actualidad es de 37,5 horas semanales-, pero esa previsión de funciones tiene poco que ver con la docencia que se desarrolla en nuestras universidades tras la adaptación al EEES ${ }^{36}$.

34 C. Rozman y J. L. Lafarga Traver (2005), «Universidad de ciencias de la salud: aspectos jurídicos de una opción innovadora», Medicina Clínica, 124 (13): págs.499-503.

35 Lo mismo se puede decir del RD 898/1985, de 30 de abril, que regula el régimen del profesorado, cuya vigencia se ha ido alargando por la falta de consenso para aprobar un nuevo estatuto del profesorado universitario.

36 La dedicación horaria semanal de dicha jornada será la siguiente: a) Seis horas semanales, como máximo, exclusivamente docentes, durante el período lectivo. b) Tres horas semanales, corno máximo, de asistencia y tutoría al alumnado durante el periodo lectivo, que podrá realizarse en la institución sanitaria concertada. c) Veinticinco horas semanales, como mínimo, de asistencia sanitaria en la correspondiente Institución. En dichas horas quedará incluida, en su caso, la docencia práctica y la función investigadora que implique actividad asistencial. La docencia práctica conllevará, en todo caso, la responsabilidad directa del profesor en el aprendizaje clínico de los alumnos que le sean asignados. d) El resto de las horas de la jornada semanal legalmente establecida, tanto en el período lectivo como en el período no lectivo, se dedicarán a la función investigadora que no implique actividad asistencial, así como, en su caso, a la atención de las necesidades de gestión y administración inherentes al cargo o puesto de trabajo que se desempeñe en los ámbitos docentes y asistencial. Los profesores asociados con plaza asistencial en los centros concertados tendrán, en su calidad de profesor, el régimen de dedicación a tiempo parcial de tres horas lectivas semanales y un número igual de horas de tutoría y asistencia a los alumnos, que se desarrollarán dentro de la jornada laboral legalmente establecida, de acuerdo con lo dispuesto en el punto dos de esta base. 
A modo de ejemplo, se recoge que en los periodos en los que la programación académica no incluya el desarrollo de las horas exclusivamente docentes, el cómputo de la jornada no será inferior a la legalmente establecida, dedicándose dichas horas a las actividades asistenciales, lo cual ahora tiene difícil encaje ya que los períodos lectivos se han modificado (en realidad fuera del mes vacacional de agosto, todo el tiempo hay actividad docente de uno u otro tipo), las tutorías se han incrementado, la atención a los alumnos es mucho más personalizada, es necesario y obligatorio formar parte de tribunales de TFGS e igualmente es obligatorio dirigir estos trabajos, con lo cual la compatibilidad de toda esta labor con el límite de horas semanales resulta muy complejo. Además, la dedicación del profesorado se fija en el art. 68 de la LOU desde el año 2012, sin distinguir si son o no vinculados, señalando en algunos casos la obligación de impartir hasta 32 créditos anuales si no han obtenido un sexenio de investigación en los últimos seis años, lo cual implica claramente más de seis horas docentes a la semana. La dedicación docente se fija con carácter general en 24 créditos (lo cual implica una media de $8 \mathrm{~h}$ semanales) siempre que se haya obtenido el último sexenio. Solo cuando se alcanza un determinado número de sexenios, esta dedicación puede reducirse a 16 créditos anuales. De hecho, para los profesores titulares y catedráticos, en este punto, ha dejado de ser de aplicación el RD 898/1985, que fijaba las obligaciones docentes en ocho horas semanales de clase y seis horas de tutoría, ya que se entiende derogado por la LOU.

Todo ello ha traído consigo un incremento de la dedicación horaria del profesorado a su actividad docente, que se agrava en el caso de los profesores vinculados, que tienen que dedicar un $75 \%$ de su jornada al ámbito asistencial, lo cual puede llevar a que mantener la calidad y la exigencia en todos los ámbitos resulte sencillamente imposible.

Además, desde el ámbito universitario el trato diferenciado a estos profesores puede generar tensiones con el resto de profesorado; y, al revés, una mayor dedicación docente generará probablemente el conflicto en el ámbito asistencial, donde la carga de trabajo es muy intensa y conviven profesionales vinculados y no vinculados. A ello hay que añadir que, en esa jornada única, el profesor vinculado tiene que dedicar tiempo a la investigación, pues su peso en las acreditaciones hasta ahora ha sido determinante y a partir de ahora seguirá siendo muy importante ${ }^{37}$.

37 Además, la Ley 14/2007, de 3 de julio, de Investigación biomédica, en sus arts. 82 y ss., establece el fomento de la actividad investigadora en todo el Sistema Nacional de Salud como elemento fundamental para el desarrollo del mismo y, en concreto, la necesidad del desarrollo de la actividad investigadora en los hospitales como núcleos vertebradores de la investigación en forma cooperativa y de red. 
Llama mucho la atención que, a pesar de que los conciertos tengan entre sus objetivos potenciar la investigación en las ciencias de la salud y la estimulación de vocaciones investigadoras, el RD 1558/86 despache la investigación con una dedicación totalmente residual ${ }^{38}$, incluyendo dentro de la dedicación asistencial la función investigadora que implique actividad asistencial y al resto de la investigación podrá dedicar unas 3 horas a la semana (no en exclusiva, sino junto a la gestión que implique su puesto). Sobra prácticamente todo comentario a esta realidad.

Una nueva regulación tendrá que fijar nuevos contenidos para la dedicación de los profesores vinculados que den respuesta a la realidad docente y asistencial vigente en estos momentos. La coordinación se hace imprescindible si no se quiere entrar en un conflicto permanente, y las comisiones mixtas no siempre han acertado con las medidas idóneas para que las funciones académicas y las asistenciales no choquen ${ }^{39}$. Por ello, una nueva normativa debería incluir normas claras de coordinación que eviten las luchas veladas sobre qué ámbito se impone sobre el otro ${ }^{40}$.

\section{DEDICACIÓN, INCOMPATIBILIDADES Y JUBILACIÓN}

Los profesores vinculados cuentan con los mismos derechos que el resto de profesorado universitario, por lo que su régimen de dedicación se ajustará a lo dispuesto en el art. 9 del RD 898/85. En esta norma, con carácter general, se da preferencia a la dedicación a tiempo completo sobre la dedicación a tiempo parcial, si bien el profesorado podrá solicitar el tipo de dedicación que quiera tener. La universidad la concederá o denegará atendiendo a las necesidades del servicio y a la disponibilidad presupuestaria. En el caso de los profesores vinculados, el régimen de dedicación implicará que en la misma jornada se desarrollarán el conjunto de sus actividades docentes, asistenciales y de investigación. El régimen de dedicación a tiempo completo comportará la

38 En su primera redacción, ni siquiera se hacía referencia a la labor investigadora, razón por la cual el TS declaró nula la base decimotercera (STS de 3 de julio de 1989), como ya se ha seńalado.

39 En otras, en cambio, el día a día ha hecho que se fijen normas para el funcionamiento, y en concreto para las sustituciones, vacaciones, licencias y permisos del personal docente. Véanse las normas aprobadas por la Comisión mixta del concierto entre la universidad de Las Palmas y el Servicio Canario de la Salud.

40 C. Rozman y J. L. Lafarga Traver, «Universidad de ciencias de la salud: aspectos jurídicos de una opción innovadora», op. cit., págs. 499-503. Estos autores ponen de relevancia como la regulación actual ha llevado a una creciente influencia de las instituciones sanitarias sobre el ámbito académico. 
exclusiva dedicación a los sistemas sanitario y docente, por lo tanto, impedirá el ejercicio de la actividad privada. En ambos casos, la duración de la jornada laboral será la legalmente establecida para el personal con plaza exclusivamente asistencial en los centros sanitarios públicos.

En cuanto al régimen de incompatibilidades, la realización de funciones docentes por el personal de las instituciones sanitarias concertados mediante contrato con la universidad como profesor asociado, requerirá el reconocimiento previo de compatibilidad por la institución sanitaria. Igualmente, el desarrollo de actividad privada por el personal que desempeñe plaza vinculada o sea contratado como profesor asociado, de acuerdo con lo dispuesto en el Real Decreto 1558/1986, precisará la correspondiente autorización de compatibilidad. En todo caso, no podrá autorizarse dicha compatibilidad para el desarrollo de actividad privada al personal que desempeńe plaza vinculada por la que perciba complemento específico o concepto equiparable, ni al personal asistencial contratado como profesor asociado, que perciba el mismo complemento retributivo por su actividad asistencial. Esta compatibilidad la otorgará el rector de la universidad si se trata de profesores titulares y catedráticos de universidad, y por la institución sanitaria, si son profesores asociados de Ciencias de la Salud. Sin embargo, estas normas generales requerirían una mayor concreción, ya que la casuística en los últimos años se ha incrementado, en función de las distintas normativas autonómicas, por lo que una regulación más detallada de las compatibilidades de los profesores vinculados facilitaría su aplicación.

Por último, me referiré al tema de la jubilación de los profesores vinculados que, en los últimos tiempos, ha dado lugar a multitud de conflictos, hasta el punto de que el TC ha tenido que pronunciarse sobre el asunto. La jubilación del personal estatutario conforme al art. 26 de la Ley 55/2003 se producirá con carácter forzoso a los sesenta y cinco años. Sin embargo, el interesado podrá solicitar voluntariamente prolongar su permanencia en el servicio activo hasta cumplir, como máximo, los setenta años de edad, siempre que quede acreditado que reúne la capacidad funcional necesaria para ejercer la profesión o desarrollar las actividades correspondientes a su nombramiento. Esta prolongación está condicionada a autorización por el servicio de salud de la Comunidad Autónoma, que la concederá o denegará en función de las necesidades de la organización articuladas en el marco de los planes de ordenación de recursos humanos. Solamente se configura con carácter obligatorio, siempre que quede acreditado que el interesado reúne la capacidad funcional necesaria para ejercer la profesión o desarrollar las actividades correspondientes a su nombramiento y así lo solicite, si en el momento de cumplir la edad de jubilación forzosa, le restan seis años o menos de cotización para causar pensión de jubilación. 
Con esta regulación en los primeros momentos, los servicios de salud autonómicos venían concediendo la prolongación de forma más o menos generalizada, pero, a partir de 2011, entre las medidas de contención del gasto, muchas CC.AA. prohibieron la posibilidad de prolongar la permanencia en el servicio activo, en este ámbito y en otros del empleo público (art. 67.3 EBEP). Esto trajo consigo que algunos profesores vinculados se encontrasen en una situación compleja, ya que en el ámbito asistencial se jubilaban, lo cual llevaba aparejada la pérdida de la vinculación de la plaza, sin embargo, en el ámbito universitario tenían derecho a jubilarse a los setenta años de acuerdo

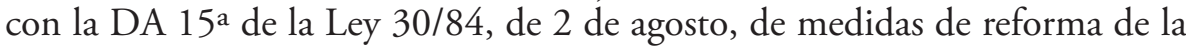
función pública.

El caso que da lugar a la STC 44/2016, de 14 de marzo, es lo bastante significativo para traerlo a colación por ser un claro exponente de la conflictividad que generan las plazas vinculadas, tanto en el ámbito universitario como en el asistencial. Un profesor titular de la universidad de Lleida venía ejerciendo plaza vinculada en el área de conocimiento de Medicina, vinculada al Instituto Catalán de la Salud (ICS). El ICS declaró su jubilación por cumplir 65 años de edad, sin embargo, el profesor entendió que podría seguir como tal en la universidad hasta cumplir 70 años y, por ello, solicitó la desvinculación de su plaza al rector. El rector dicta resolución de 30 de septiembre de 2005 rechazando su solicitud y declarando también su jubilación en el ámbito universitario, resolución que da lugar a todo el periplo judicial que se desarrolla en los once años siguientes. El TSJ de Cataluña rechaza su recurso por sentencia de 20 de enero de 2009 y el afectado recurre al TS, que finalmente le da la razón en su sentencia de 12 de diciembre de $2011^{41}$. La universidad de Lleida fundamentó la jubilación en que la dedicación del recurrente era a tiempo parcial y que había comunicado al actor que la prórroga del servicio activo hasta los setenta años requería mantener su vinculación profesional con el ICS, donde había sido jubilado el 5 de julio de 2005, defendiendo la obligación de la universidad de desvincular la plaza. El TS estima el recurso de casación fundando su resolución en los siguientes argumentos, que pasamos a sintetizar:

- La pertenencia al cuerpo de profesores titulares de universidad implica el derecho a no jubilarse forzosamente por razón de edad hasta cumplir los setenta años, conforme a la disposición adicional décimo quinta de la Ley 30/1984.

- Ni del art. 61 de la LOU ni del art. 105 de la LGS resulta lo contrario. Entendiendo el TS que las singularidades de los profesores vinculados frente

41 RJ 2716/2012. 
al resto de profesores son mínimas y limitadas por la legislación (exigencia del título de médico especialista para los aspirantes a las plazas, cumplir las exigencias que en cuanto a su cualificación asistencial se determinen reglamentariamente, la especial composición de las comisiones de acceso a los cuerpos docentes y la necesidad de evaluación de la labor asistencial).

- El Real Decreto 1558/1986, cuando regula el régimen de los conciertos a suscribir entre las universidades y las instituciones sanitarias respecto de las plazas vinculadas, se preocupa porque en ellos se salvaguarden los derechos del profesorado universitario.

- Entiende el TS que existe un «derecho inherente a la condición de funcionario de los cuerpos docentes universitarios de no jubilarse forzosamente por edad antes de cumplir los setenta años». Y dice expresamente que «no hay norma legal que disponga para los profesores universitarios que desempeñan plazas vinculadas un régimen de jubilación diferente al de los demás».

- Remata su argumentación señalando que la naturaleza de las plazas vinculadas no afecta al régimen de jubilación porque «ni la legislación universitaria ni la sanitaria han previsto para quienes las desempeñan un régimen de jubilación diferente al del resto del profesorado universitario. En segundo lugar, porque cabe desvincularlas. Y, en tercer lugar, porque siendo cierto que se es profesor titular de universidad de una determinada área de conocimiento, también lo es que los departamentos llamados a organizar la docencia disponen de margen suficiente para aprovechar de manera satisfactoria el personal».

Como en el momento en que se dicta la sentencia el recurrente ya había cumplido los 70 años, además de anular el acto, la sentencia obliga a la universidad de Lleida a indemnizarle por el importe resultante de la diferencia existente entre los ingresos que ha percibido en concepto de pensión por jubilación y los que le habrían correspondido como profesor titular en activo.

Así las cosas, y con la rotundidad con la que se había expresado el TS, lo normal es que esta sentencia hubiese puesto punto final al asunto, pero lo cierto es que no fue así. La universidad de Lleida, en total desacuerdo con el pronunciamiento, presentó un recurso de amparo por vulneración del derecho fundamental a la tutela judicial efectiva y a la autonomía universitaria, que dará lugar a una sentencia del TC rechazando el amparo. El TC niega además que como persona jurídica pública la universidad pueda alegar el derecho fundamental a la tutela judicial efectiva, pero admite el recurso por vulneración de la autonomía universitaria. En el pronunciamiento, tras analizar el alcance de la autonomía universitaria, haciendo un recorrido por su jurisprudencia previa, alcanza la conclusión de que: 
No cabe defender que, en aras de garantizar la autonomía universitaria, las universidades puedan modular la edad de jubilación del profesorado adscrito a plazas vinculadas, es decir, puedan ajustar en su interés la aplicación del régimen funcionarial de jubilación forzosa, mediante un instrumento administrativo como es la decisión de desvincular o no una determinada plaza previamente vinculada, una decisión que no está sujeta a ningún elemento reglado y que no está prevista para que las comisiones mixtas modulen el régimen de jubilación aplicable al profesorado, porque este régimen solo puede venir determinado por la ley.

Con este pronunciamiento del TC sí que se pone fin a este caso, en el que el interesado se ha visto inmerso en un proceso judicial que ha durado once años.

En relación con la jubilación de los profesores vinculados y de los facultativos en general, hay que señalar que muchas de las CC. AA. están en estos momentos flexibilizando la posibilidad de prolongación de la edad de jubilación hasta los setenta años, volviendo a la situación previa a los ajustes en el empleo público derivados de la crisis económica. Además, alguna normativa autonómica, como la Ley 9/2010 de Cantabria (ya mencionada), prevé que, alcanzada la edad legal de jubilación forzosa prevista para el personal estatutario en la legislación vigente, el titular de plaza vinculada cese en la jefatura de servicio o de sección que, en su caso, ostentase, continuando vinculado a una plaza asistencial básica.

\section{REFLEXIONES FINALES}

La necesidad de reforma de la normativa reguladora del régimen jurídico de las plazas de profesores universitarios vinculadas a las instituciones sanitarias está fuera de toda duda. Treinta años de aplicación del RD 1558/1986, la conflictividad que ha generado y la jurisprudencia dictada en la materia, creo que permiten afrontar este reto con ciertas ventajas frente a la primera regulación, por lo que el Ministerio de Sanidad y el de Educación, junto con la CRUE, deberían abordar la cuestión. Ahora bien, que sea necesario modificar y adaptar la normativa a todos los cambios producidos tanto en el ámbito docente, como en el asistencial no significa que haya que cambiar el modelo de formación, porque creo que es muy importante señalar que este modelo ha formado a nuestros facultativos al más alto nivel.

Los últimos diez años tampoco deben ser determinantes a la hora de tomar decisiones, ya que han confluido demasiadas circunstancias externas que han puesto en jaque al modelo. La superación de la crisis económica, aunque sea lenta, volverá a atenuar algunos de los problemas surgidos, como la falta 
de convocatoria de plazas, y el nuevo modelo de acreditación debería permitir que la obtención de acreditaciones se normalizase en este campo del conocimiento con una adecuada evaluación de la labor asistencial. En todo caso, tal vez el envejecimiento acumulado en las plantillas debería llevar a algún tipo de solución ad hoc en lo que se refiere a la convocatoria de plazas para que la calidad de la docencia no se resienta y sea posible un relevo generacional.

El tema de la equivalencia entre categorías docentes y asistenciales es complejo por las razones expuestas y, sobre todo, por la confluencia de dos modelos de acceso/provisión muy diferentes, en el ámbito docente y en el ámbito asistencial. En todo caso, sí se podría avanzar en previsiones generales que facilitasen esa equivalencia en algunos supuestos, ya que indudablemente disminuirían las disfunciones entre el ámbito docente y asistencial. Está claro que cuando coinciden los objetivos docentes y asistenciales y las universidades y entidades sanitarias logran acuerdos, el funcionamiento y los resultados son más satisfactorios.

En cuanto a las modificaciones a abordar, creo que es muy importante reconocer dentro de la dedicación del profesor vinculado mayor tiempo para la labor investigadora, no solo por el hecho de que así se facilitaría la obtención de las acreditaciones, sino también porque muchos de nuestros hospitales universitarios deberían intensificar su participación en la $\mathrm{I}+\mathrm{D}+\mathrm{i}$ al máximo nivel. La parte asistencial es clave cuando se habla de sanidad, pero la labor investigadora no se debería arrinconar por muy importante que sea reducir las listas de espera.

Hemos formado unos profesionales excelentes y ahora deberíamos tratar que todo su talento revierta en beneficio de la sociedad y que las generaciones más jóvenes de profesionales no consideren la carrera académica e investigadora como un imposible. Por ello, hacer más atractiva la misma debe ser uno de los objetivos de la reforma. 
